\title{
Prevalence and risk factors for hypokalemia in patients scheduled for laparoscopic colorectal resection and its association with post-operative recovery
}

Qianqian Zhu ${ }^{1,2+}$, Xianlong $\mathrm{Li}^{1 \dagger}$, Fang Tan ${ }^{2 \dagger}$, Yingqing Deng ${ }^{1}$, Chulian Gong ${ }^{1}$, Jingping Hu${ }^{1}$, Pinjie Huang ${ }^{1 *}$ and Shaoli Zhou ${ }^{1 *}$

\begin{abstract}
Background: Perioperative serum potassium levels are closely associated with postoperative clinical outcomes after gastrointestinal surgery. The aim of our retrospective study was to identify the prevalence and risk factors for preoperative hypokalemia (before pneumoperitoneum) and to evaluate the influence of preoperative hypokalemia on the recovery of postoperative gastrointestinal function.

Methods: In this retrospective study, patients scheduled for laparoscopic colorectal resection from November 11 2014 to October 20 2016, were considered for inclusion. A blood potassium level between 3.5 and $5.5 \mathrm{mmol} / \mathrm{L}$ was defined as normal, with levels between 3.0 to $3.5 \mathrm{mmol} / \mathrm{L}, 2.5$ to $3.0 \mathrm{mmol} / \mathrm{L}$ and $<2.5 \mathrm{mmol} / \mathrm{L}$ considered as slight, moderate, and severe level of hypokalemia. The factors including age, gender, ASA grade, BMI, hypertension, diabetes, anti-hypertension drugs, lactose oral soluble, oral cathartics, oral cathartics, cathartic enemas, and blood potassium level before gastrointestinal preparation which might be associated with blood potassium level before pneumoperitoneum were analysed. The time to postoperative first flatus (FFL) and first feces (FFE) was compared between patients with and without hypokalemia.

Results: The final analysis was based on the data of 108 patients. Hypokalemia was identified in $70.37 \%$ patients, with the following distribution of blood potassium levels before pneumoperitoneum: slight, 49 (45.37\%) patients; moderate, 23 (21.30\%); and severe, 4 (3.70\%) patients. Hypokalemia was significantly associated with hypertension and the use of $\geq 2$ types of oral cathartics for preoperative gastrointestinal preparation. With treatment, potassium levels recovered to normal levels in all patients within $48 \mathrm{~h}$ postoperatively. Hypokalemia was associated with a longer postoperative time to first feces, compared to patients with a normal potassium level before pneumoperitoneum.
\end{abstract}

Conclusions: Our findings underlie the importance of early monitoring and management of serum potassium levels in these patients.

Keywords: Hypokalemia, Gastrointestinal preparation, Risk factors

\footnotetext{
* Correspondence: hpjie@126.com; shaolizhou@139.com

†Qianqian Zhu, Xianlong Li and Fang Tan contributed equally to this work.

'Department of Anesthesiology, The Third Affiliated Hospital of Sun Yat-Sen

University, 600th Tianhe Road, Tianhe District, 510360 Guangzhou City,

People's Republic of China

Full list of author information is available at the end of the article
}

(c) The Author(s). 2018 Open Access This article is distributed under the terms of the Creative Commons Attribution 4.0 International License (http://creativecommons.org/licenses/by/4.0/), which permits unrestricted use, distribution, and reproduction in any medium, provided you give appropriate credit to the original author(s) and the source, provide a link to the Creative Commons license, and indicate if changes were made. The Creative Commons Public Domain Dedication waiver (http://creativecommons.org/publicdomain/zero/1.0/) applies to the data made available in this article, unless otherwise stated. 


\section{Background}

Perioperative (including before,during and after surgery) serum potassium levels are closely associated with postoperative clinical outcomes after gastrointestinal surgery [1], including the recovery of gastrointestinal function, the risk for acute kidney injury and adverse cardiovascular events, as well as in-hospital mortality [2-4]. Hypokalemia occurs frequently, being identified in over one fifth of hospitalized patients [5].

Several factors are believed to be involved in the development of hypokalemia [6], including inadequate intake, excessive potassium loss and impairment in the distribution mechanisms of potassium. Although diuretic therapy is considered to be the main risk factor for hypokalemia, diarrhea, vomiting and gastrointestinal preparation prior to surgery may also be contributing factors [7]. For gastrointestinal preparation prior to abdominal surgery, dietary restrictions, oral cathartics, cathartic enemas, and colon-cleansing agents are used to achieve ideal preparation for both surgery and anesthesia. As a result, perioperative hypokalemia is a common finding among these patients, which could affect postoperative gastrointestinal function [8, 9]. However, the prevalence and risk factors for perioperative hypokalemia in these patients remains unclear, including the influence of preoperative (before pneumoperitoneum) hypokalemia on the postoperative recovery of gastrointestinal function. Therefore, the aim of our retrospective study was to identify the prevalence and risk factors for preoperative hypokalemia and to evaluate the influence of preoperative hypokalemia on the recovery of postoperative gastrointestinal function.

\section{Methods}

\section{Patients and data collection}

From November 112014 to October 20 2016, a total of 122 patients scheduled for laparoscopic colorectal resection at the Third Affiliated Hospital of Sun Yat-sen University were considered for inclusion. The clinical data of prospective patients was reviewed from medical records to include patients $\geq 18$-years-old, with an American Society of Anesthesiologists (ASA) Physical grade I/II/III physical status. Patients with a history of familial hypokalemia, pre-existing kidney disease and whose blood potassium levels were not obtained before pneumoperitoneum were excluded.

\section{Ethical standard}

This study was approved by the Ethics Committee of the Third Affiliated Hospital of Sun Yat-sen University and was carried out in compliance with the Helsinki Declaration. The requirement for informed consent was waived because of the retrospective nature of the study and our use of anonymized data.

\section{Criteria of hypokalemia}

In agreement with previous studies, a blood potassium level between 3.5 and $5.5 \mathrm{mmol} / \mathrm{L}$ was defined as normal, with levels between 3.0 to $3.5 \mathrm{mmol} / \mathrm{L}, 2.5$ to $3.0 \mathrm{mmol} / \mathrm{L}$ and $<2.5 \mathrm{mmol} / \mathrm{L}$ considered as slight, moderate, and severe level of hypokalemia [8].

\section{Statistical analysis}

Quantitative variables with a normal distribution are reported as the mean \pm standard deviation (SD). The one-sample Kolmogorov-Smirnov Test was used to test the normality of the distribution of quantitative data, the Student's $t$-test was used to compare: normally distributed variables between the two groups, and the Mann-Whitney $\mathrm{U}$ test was used for non-normally distributed data. Categorical data were compared using the chi-squared or Fisher's exact tests. A multivariable logistic regression analysis (forward regression:LR method) was used to determine risk factors for hypokalemia. Differences were considered significant when the two-tailed $p$ values were $<0.05$. SPSS 19.0 software (SPSS Inc., Chicago, IL) was used to perform statistical analyses.

\section{Results}

The final analysis was based on the data of 108 patients, with at least one blood potassium level obtained during the peri-anesthesia period (Fig. 1). There were 76 patients with hypokalemia. Hypokalemia was identified in $70.37 \%$ patients, with the following distribution of blood potassium levels before pneumoperitoneum: slight, 49(45.37\%) patients; moderate, 23(21.30\%); and severe, 4 (3.70\%) patients. The distribution of hypokalemia severity at the different time points of measurement is summarized in Table 1. To identify the preoperative factors associated with hypokalemia before pneumoperitoneum, the 108 patients were classified into the normal potassium level group (non-hypokalemia group) or the hypokalemia group. The characteristics of the 108 patients are summarized in Table 2 .

There were no significant differences between patients with or without hypokalemia with regard to age, sex, body mass index (BMI), and ASA grade. However, the incidence rate of hypertension was greater in the hypokalemia (44.7\%) than the non-hypokalemia (18.8\%) group $(p=0.011)$. In terms of gastrointestinal preparation, the proportion of patients in whom $\geq 2$ types of oral cathartics were used was greater in the hypokalemia $(63.2 \%)$ than that in the non-hypokalemia $(34.4 \%)$ group $(p=0.006)$. With regard to the use of cathartic enemas, although more patients used lactose oral soluble or $\geq 2$ types of enemas than in the non-hypokalemia group, this between-group difference was not significant. The variables which were statistical different between hypokalemia and non-hypokalemia group were included in 


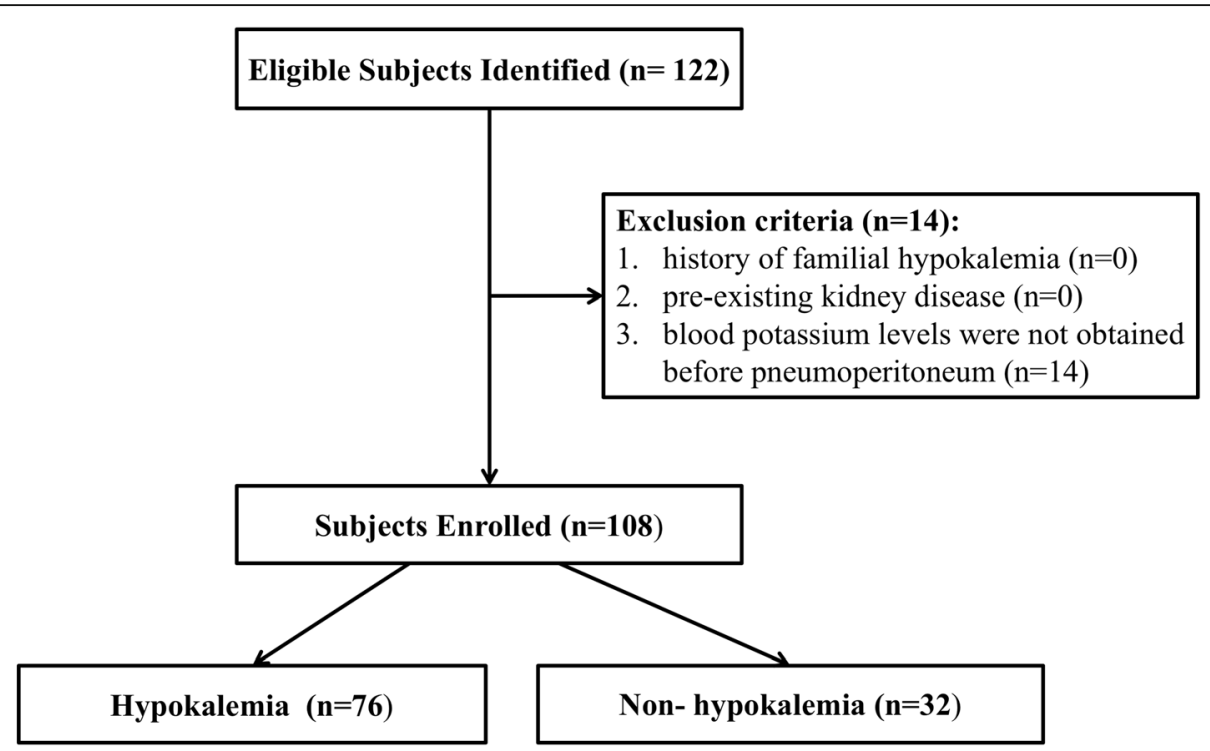

Fig. 1 The flow chart of inclusion and exclusion process

multivariable model. The multivariable logistic regression analysis (forward regression: LR method) was used to determine risk factors for hypokalemia. On multivariable logistic regression analysis, hypertension (odds ratio (OR), 4.067; 95\% confidence interval (CI), 1.434-11.532) and use of $\geq 2$ types of oral cathartics (OR, 3.743; 95\%CI, 1.508-9.296) were significantly related with hypokalemia prior to pneumoperitoneum (Table 3). Following intraoperative treatment, only 6 patients presented with slight hypokalemia at $24 \mathrm{~h}$ post-surgery (Table 1 ).

With regard to the association between blood potassium level prior to pneumoperitoneum and postoperative gastrointestinal function, the time to postoperative first flatus was not significantly different between patients with a normal blood potassium level (55.53 \pm $27.34 \mathrm{~h})$ and those with hypokalemia $(58.38 \pm 26.05 \mathrm{~h}$, $p=0.618)$. However, the time to first feces was significantly longer among patients with hypokalemia (97.71 \pm $50.83 \mathrm{~h}$ ) than in those with a normal blood potassium level $(79.81 \pm 37.04 \mathrm{~h}, p=0.045)$.

Table 1 The distributions of hypokalemia in different time points

\begin{tabular}{llll}
\hline & $\begin{array}{l}\text { Before gastrointestinal } \\
\text { preparation (108) }\end{array}$ & $\begin{array}{l}\text { Before } \\
\text { pneumoperitoneum } \\
(108)\end{array}$ & $\begin{array}{l}\text { 24 h after } \\
\text { surgery (108) }\end{array}$ \\
\hline $\begin{array}{l}\text { Slight } \\
\text { hypokalemia }\end{array}$ & $18(16.67 \%)$ & $49(45.37 \%)$ & $6(5.56 \%)$ \\
$\begin{array}{l}\text { Moderate } \\
\text { hypokalemia }\end{array}$ & 0 & $23(21.30 \%)$ & 0 \\
$\begin{array}{l}\text { Severe } \\
\text { hypokalemia }\end{array}$ & 0 & $4(3.7 \%)$ & 0 \\
\hline
\end{tabular}

Table 2 Characteristics of 108 patients

\begin{tabular}{|c|c|c|c|}
\hline Characteristic & $\begin{array}{l}\text { Hypokalemia } \\
(n=76)\end{array}$ & $\begin{array}{l}\text { Non- hypokalemia } \\
(n=32)\end{array}$ & $P$-value \\
\hline Age & $60.34 \pm 9.89$ & $57.75 \pm 13.13$ & 0.107 \\
\hline Gender (female) & $41(53.9 \%)$ & $11(34.4 \%)$ & 0.063 \\
\hline BMI $\left(\mathrm{kg} / \mathrm{m}^{2}\right)$ & $22.93 \pm 3.26$ & $22.48 \pm 2.92$ & 0.616 \\
\hline ASA grade & & & 0.391 \\
\hline I & $5(6.6 \%)$ & $1(3.1 \%)$ & \\
\hline$\|$ & $58(76.3 \%)$ & $28(87.5 \%)$ & \\
\hline III & 13(17.1\%) & $3(9.4 \%)$ & \\
\hline Hypertension & $34(44.7 \%)$ & $6(18.8 \%)$ & 0.011 \\
\hline \multicolumn{4}{|l|}{ Anti-hypertension drugs } \\
\hline Calcium channel blockers & 16 & 2 & \\
\hline$\beta$-receptor blocker & 3 & 1 & \\
\hline ACEl and ARB & 2 & 0 & \\
\hline Thiazide & 2 & 0 & \\
\hline Diabetes & $9(11.8 \%)$ & $3(9.4 \%)$ & 0.97 \\
\hline Lactose oral soluble & $30(39.5 \%)$ & $9(28.1 \%)$ & 0.262 \\
\hline Oral cathartics ( $\geq 2$ types) & $48(63.2 \%)$ & $11(34.4 \%)$ & 0.006 \\
\hline $\begin{array}{l}\text { Cathartic enemas } \\
\text { ( } \geq 2 \text { types) }\end{array}$ & $47(61.8 \%)$ & 19(59.4\%) & 0.810 \\
\hline $\begin{array}{l}\text { Hypokalemia before } \\
\text { gastrointestinal preparation }\end{array}$ & 15(19.7\%) & $3(9.4 \%)$ & 0.187 \\
\hline Time to first flatus (h) & $58.38 \pm 26.05$ & $55.53 \pm 27.34$ & 0.618 \\
\hline Time to first feces (h) & $97.71 \pm 50.83$ & $79.81 \pm 37.04$ & 0.045 \\
\hline
\end{tabular}

Notes: Data were presented by mean \pm SD and percentages. The onesample Kolmogorov-Smirnov Test was used to test the normality of the distribution of quantitative data. Normally distributed variables were compared using Student's $t$-test, non-normally distributed variables using Mann-Whitney $\mathrm{U}$ test, and categorical data using the chi-squared or Fisher's exact tests; $P$-value $<0.05$ was considered significant Abbreviations: BMI body mass index, ASA American Society of Anesthesiologists, $A C E$ angiotensin converting enzyme inhibitors, $A R B$ angiotensin receptor blocker 
Table 3 Multivariable logistic regression results for Hypokalemia patients

\begin{tabular}{lllll}
\hline Factors & OR & $95 \% \mathrm{Cl}$ & P-value \\
\cline { 3 - 4 } & & Lower & Upper & \\
\hline Hypertension & 4.067 & 1.434 & 11.532 & 0.008 \\
$\begin{array}{l}\text { Oral Cathartics } \\
\text { ( } \geq 2 \text { types) }\end{array}$ & 3.743 & 1.508 & 9.296 & 0.004 \\
\hline
\end{tabular}

Notes: Multivariable logistic regression analysis (forward LR method) was used to determine risk factors for hypokalemia; $P<0.05$ was considered to be statistically significant

Abbreviations: $O R$ odds ratio, $\mathrm{Cl}$ confidence interval

\section{Discussion}

In our study group, the incidence rate of hypokalemia was $70.37 \%$, with $3.70 \%$ patients presenting with severe hypokalemia in the peri-anesthesia period. Hypokalemia was significantly associated with hypertension and the use of $\geq 2$ types of oral cathartics for preoperative gastrointestinal preparation. With treatment (potassium chloride was intravenous infusion until potassium level reach $3.5 \mathrm{mmol} / \mathrm{L}$ during surgery, oral potassium $1 \mathrm{~g} /$ day in inpatient ward), potassium levels recovered to normal levels in all patients within $48 \mathrm{~h}$ postoperatively. Hypokalemia was associated with a longer postoperative time to first feces, compared to patients with a normal potassium level before pneumoperitoneum.

The prevalence of rate of hypokalemia in our study group of $70.37 \%$ was higher than the rate previously reported for a general group of hospitalized patients [5]. Hypokalemia is common in hospitalized patients. Various factors can contribute to hypokalemia, including insufficient intake, excessive loss of potassium and inadequate potassium distribution. Specifically for laparoscopic resection, the strict gastrointestinal preparation required can lead to hypokalemia. Our findings are in agreement with a previous study which demonstrated that the development of hypokalemia was a common complication of polyethylene glycol-based preoperative bowel preparation [10]. Though the polyethylene glycol is the most frequently used solutions for bowel preparation, other oral cathartics were also used. In the present study, patients with constipation were more prone to take other cathartics like lactulose or mannitol before bowel preparation with polyethylene glycol, even early when admitted to hospital. Mannitol administration could also induce hypokalemia [11]. We also identified that patients in whom $\geq 2$ types of oral cathartics were used were more likely to develop hypokalemia. Therefore, potassium monitor should be recommended for these patients early even before gastrointestinal preparation.

In addition to potassium loss through the gastrointestinal tract, the use of diuretics can further contribute to hypokalemia [12]. Thiazide, which is often prescribed to patients with cardiovascular disease, such as hypertension, can induce hypokalemia through this mechanism [13]. In our study, the prevalence of hypertension was greater among patients with hypokalemia than without. However, the use of anti-hypertension drugs, including thiazide, was comparable between the two groups, a finding which might be attributed to the limited number of patients. Of note, however, hypertension was identified as an independent risk factor for hypokalemia on multivariable analysis in our study. Hypokalemia in hypertension might result from an increase in urinary loss of potassium with the use of anti-hypertensive drugs, such a thiazide mentioned above, which are used to block the increased reabsorption of sodium associated with hypertension [14]. Of note, although hyperkalemia has been described as an adverse outcome of the use of angiotensin-converting-enzyme inhibitors (ACEIs) and angiotensin receptor blockers (ARBs), two types of anti-hypertensive drugs, hypokalemia has also been described, particularly in those patients on dual therapy [15].

Although hypokalemia is often asymptomatic, it remains a risk factor of gastrointestinal disorders and serious perioperative and postoperative arrhythmia [16-19]. Hypokalemia might also contribute to delayed recovery from anesthesia [20], as well as increasing the length of hospital stay and the risk for all-cause and cardiovascular-related mortality $[5,21]$. Among our study group, the time to first feces was significantly longer among patient with than without hypokalemia. Therefore, maintaining normal potassium after laparotomy would be important to lower the risk for gastrointestinal mortality and to improve postoperative recovery. In fact, in the "enhanced recovery after surgery" (ERAS) protocol, prophylactic potassium administration is recommended [22]. Another study even demonstrated that the prevention of hypokalemia prior to admission was effective in enhancing the recovery of patients undergoing open abdominal surgeries [8].In our study group, the prevalence of hypokalemia prior to gastrointestinal preparation was not high $(16.67 \%)$, with the level of hypokalemia in these patients being slight. By contrast, the prevalence of hypokalemia increased to $70.37 \%$ after gastrointestinal preparation, with $3.70 \%$ of patients presenting severe hypokalemia. Previous studies demonstrated that early serum potassium monitoring allowed for early correction of hypokalemia and effective recovery from surgery $[4,8]$. Based on this evidence, early potassium administration has been strongly recommended for patients scheduled for elective laparoscopic colorectal resection under strict monitoring, especially during the period of bowel preparation. Whether hypertensive patients were more like to develop hypokalemia during gastrointestinal preparation remains to be confirmed and explored in future studies.

There are several limitations of the present study which need to be declared. First, because of the limited number of patients and selection from a single center, results, including the prevalence of peri-anesthesia hypokalemia, will need to be confirmed in larger, multi-center, cohort 
studies. Second, due to the retrospective nature of our study, reasons for different bowel preparation used were not clear, and might have biased our results. Third, detailed information, such as the oral intake of potassium and ambulation status of patients, could not be extracted from medical records and, again, might have biased our results. Fourth, the parameters including the time to postoperative first flatus and the time to first feces used to determine the gastrointestinal motility are clinical assessment but not the golden standard. Scintigraphic recording should be used in future study.

\section{Conclusions}

Our study demonstrated the high prevalence of hypokalemia and severe hypokalemia during the peri-anesthesia period in patients who underwent laparoscopic colorectal resection. Hypokalemia before pneumoperitoneum was associated with a longer time to first feces postoperatively. Our findings underlie the importance of early monitoring and management of serum potassium levels in these patients.

\section{Abbreviations}

ACEls: angiotensin-converting-enzyme inhibitors; ARBs: angiotensin receptor blockers; ASA: American Society of Anesthesiologists; BMI: body mass index; $\mathrm{Cl}$ : confidence interval; ERAS: enhanced recovery after surgery; FFE: first feces; FFL: first flatus; OR: odds ratio; SD: mean \pm standard deviation

\section{Funding}

The present study was supported by National Natural Science Foundation of China (No. 81600507 and No. 81501693) and Natural Science Foundation of Guangdong Province (No. 2016A030313232).

\section{Availability of data and materias}

The datasets used and analysed during the current study are available from the corresponding author on reasonable request.

\section{Author contributions}

$\mathrm{QZ}, \mathrm{SZ}$ and PH designed the study. The data collected and analyzed by XL, FT, YD, CG and JH. The manuscript was drafted by QZ. SZ and PH critically reviewed and edited the manuscript. All authors read and approved the final manuscript.

\section{Ethics approval and consent to participate}

This study was approved by the Ethics Committee of the Third Affiliated Hospital of Sun Yat-sen University and was carried out in compliance with the Helsinki Declaration. The requirement for informed consent was waived because of the retrospective nature of the study and our use of anonymized data.

\section{Consent for publication}

Not applicable.

\section{Competing interests}

The authors have no conflicts of interest in this study.

\section{Publisher's Note}

Springer Nature remains neutral with regard to jurisdictional claims in published maps and institutional affiliations.

\section{Author details}

'Department of Anesthesiology, The Third Affiliated Hospital of Sun Yat-Sen University, 600th Tianhe Road, Tianhe District, 510360 Guangzhou City, People's Republic of China. 'Department of Anesthesiology, The Seventh Affiliated Hospital of Sun Yat-Sen University, Shenzhen City, People's Republic of China.
Received: 17 May 2018 Accepted: 27 September 2018

Published online: 19 October 2018

\section{References}

1. Arora P, Pourafkari L, Visnjevac O, Anand EJ, Porhomayon J, Nader ND. Preoperative serum potassium predicts the clinical outcome after noncardiac surgery. Clin Chem Lab Med. 2017;55(1):145-53.

2. Cheungpasitporn W, Thongprayoon C, Kittanamongkolchai W, Sakhuja A, Mao MA, Erickson SB. Impact of Admission Serum Potassium on Mortality in Patients with Chronic Kidney Disease and Cardiovascular Disease. OJM. 2017.

3. Park S, Baek SH, Lee SW, Lee A, Chin HJ, Na KY, Kim YS, Chae DW, Han JS, Kim S. Elevated baseline potassium level within reference range is associated with worse clinical outcomes in hospitalised patients. Sci Rep. 2017;7(1):2402.

4. Lu G, Xu L, Zhong Y, Shi P, Shen X. Significance of serum potassium level monitoring during the course of post-operative rehabilitation in patients with hypokalemia. World J Surg. 2014;38(4):790-4.

5. Greco A, Rabito G, Pironi M, Bissig M, Parlato S, Andreocchi L, Bianchi G, Poretti Guigli M, Llamas M, Monotti R, et al. Hypokalaemia in hospitalised patients. Swiss Med Wkly. 2016;146:w14320.

6. Lindeman RD. Hypokalemia: causes, consequences and correction. Am J Med Sci. 1976;272(1):5-17.

7. Kamolpornwijit W, Phupong V. Oral potassium chloride and oral rehydration solution supplement to prevent hypokalemia in sodium phosphate regimen for bowel preparation prior to gynecological laparoscopic surgery. J Med Assoc Thail. 2008;91(5):615-8.

8. Lu G, Yan Q, Huang Y, Zhong Y, Shi P. Prevention and control system of hypokalemia in fast recovery after abdominal surgery. Curr Ther Res Clin Exp. 2013;74:68-73.

9. van Bree SH, Vlug MS, Bemelman WA, Hollmann MW, Ubbink DT, Zwinderman AH, de Jonge WJ, Snoek SA, Bolhuis $K$, van der Zanden E, et al. Faster recovery of gastrointestinal transit after laparoscopy and fast-track care in patients undergoing colonic surgery. Gastroenterology. 2011;141(3):872-80 e871-874.

10. Ho JM, Juurlink DN, Cavalcanti RB. Hypokalemia following polyethylene glycol-based bowel preparation for colonoscopy in older hospitalized patients with significant comorbidities. Ann Pharmacother. 2010;44(3): 466-70.

11. Bilotta F, Giovannini F, Aghilone F, Stazi E, Titi L, Zeppa IO, Rosa G. Potassium sparing diuretics as adjunct to mannitol therapy in neurocritical care patients with cerebral edema: effects on potassium homeostasis and cardiac arrhythmias. Neurocrit Care. 2012;16(2):280-5.

12. Rastegar A, Soleimani M. Hypokalaemia and hyperkalaemia. Postgrad Med J. 2001;77(914):759-64.

13. Rodenburg EM, Visser LE, Hoorn EJ, Ruiter R, Lous JJ, Hofman A, Uitterlinden AG, Stricker BH. Thiazides and the risk of hypokalemia in the general population. J Hypertens. 2014;32(10):2092-7 discussion 2097.

14. Lim S. Approach to hypokalemia. Acta Med Indones. 2007;39(1):56-64

15. Heerspink HJ, Gao P, de Zeeuw D, Clase C, Dagenais GR, Sleight $P$, Lonn E, Teo KT, Yusuf S, Mann JF. The effect of ramipril and telmisartan on serum potassium and its association with cardiovascular and renal events: results from the ONTARGET trial. Eur J Prev Cardiol. 2014;21(3): 299-309.

16. Podrid PJ. Potassium and ventricular arrhythmias. Am J Cardiol. 1990;65(10): 33E-44E discussion 52E.

17. Lopez-Herce J, Santiago MJ, Sanchez C, Mencia S, Carrillo A, Vigil D. Risk factors for gastrointestinal complications in critically ill children with transpyloric enteral nutrition. Eur J Clin Nutr. 2008;62(3):395-400.

18. Sanjay OP. Pre-operative serum potassium levels and peri-operative outcomes in patients undergoing cardiac surgery. Indian J Clin Biochem. 2004;19(1):40-4.

19. Wahr JA, Parks R, Boisvert D, Comunale M, Fabian J, Ramsay J, Mangano DT. Preoperative serum potassium levels and perioperative outcomes in cardiac surgery patients. Multicenter study of perioperative ischemia research group. JAMA. 1999;281(23):2203-10.

20. More $\mathrm{P}$, Laheri $\mathrm{W}$, Waigankar $\mathrm{T}$, Wagh C. Delayed recovery from anaesthesia in a patient with optimised hypothyroidism and incidental hypokalemia. J Clin Diagn Res. 2015;9(1):UD06-7. 
21. Lee S, Kang E, Yoo KD, Choi Y, Kim DK, Joo KW, Yang SH, Kim YL, Kang SW, Yang CW, et al. Lower serum potassium associated with increased mortality in dialysis patients: a nationwide prospective observational cohort study in Korea. PLoS One. 2017:12(3):e0171842.

22. Boersema GS, van der Laan L, Wijsman JH. A close look at postoperative fluid management and electrolyte disorders after gastrointestinal surgery in a teaching hospital where patients are treated according to the ERAS protocol. Surg Today. 2014;44(11):2052-7.

Ready to submit your research? Choose BMC and benefit from:

- fast, convenient online submission

- thorough peer review by experienced researchers in your field

- rapid publication on acceptance

- support for research data, including large and complex data types

- gold Open Access which fosters wider collaboration and increased citations

- maximum visibility for your research: over $100 \mathrm{M}$ website views per year

At $\mathrm{BMC}$, research is always in progress.

Learn more biomedcentral.com/submissions 